Pacific Journal of 


\title{
REAL PARTS OF UNIFORM ALGEBRAS ON THE CIRCLE
}

\author{
W. P. Novinger
}

This paper is about uniform algebras on the unit circle $\Gamma$ in the complex plane and specifically with the spaces of real parts of such algebras. The major portion of the paper is devoted to proving that if $A$ is the disc algebra on $\Gamma$ and $B$ is any uniform algebra on $\Gamma$ such that $\operatorname{Re} A \subset \operatorname{Re} B$, then either $B=C(\Gamma)$ or else $B=A \circ \Phi(=\{f \circ \Phi: f \in A\})$ for some homeomorphism $\Phi$. We also show that any homeomorphism $\Phi$ for which $\operatorname{Re} A \subset \operatorname{Re} A \circ \Phi$ must be absolutely continuous.

By a uniform algebra on $\Gamma$ we mean a subalgebra of $C(\Gamma)$ which is closed in the supremum norm, separates points, and contains the constant functions. The canonical example of a uniform algebra on $\Gamma$ (other than $C(\Gamma)$, of course) is the disc algebra $A$ : those members of $C(\Gamma)$ which have continuous extensions to the closure of the unit disc $U$ so as to be analytic in $U$. In a recent paper [3], John M. F. O'Connell sets forth some of the consequences of assuming the relationship $\operatorname{Re} A=\operatorname{Re} B$, where $\operatorname{Re} A$ and $\operatorname{Re} B$ denote the spaces of real parts of the functions in $A$ and $B$ respectively. O'Connell shows that when $\operatorname{Re} A=\operatorname{Re} B$, there is a homeomorphism $\Phi$ of $\Gamma$ onto $\Gamma$ such that $B=A \circ \Phi$, and that any homeomorphism $\Phi$ for which $\operatorname{Re} A=\operatorname{Re} A \circ \Phi$ is necessarily absolutely continuous. Thus our main results, as stated in the opening paragraph, represent generalizations of O'Connell's work to settings in which only the inclusions $\operatorname{Re} A \subset \operatorname{Re} B$ and $\operatorname{Re} A \subset \operatorname{Re} A \circ \Phi$ are assumed.

THeOREM 1. Let $A$ be the disc algebra on $\Gamma$ and $B$ be any uniform algebra on $\Gamma$ such that $\operatorname{Re} A \subset \operatorname{Re} B$. Then either $B=C(\Gamma)$ or there is a homeomorphism $\Phi$ of $\Gamma$ onto $\Gamma$ such that $B=A \circ \Phi$.

As the proof is rather lengthy, we have chosen to proceed through a sequence of three lemmas. The last of these is perhaps interesting in its own right and is suggested by work that appears in O'Connell's paper. The first lemma is also due to O'Connell. $Z$ will be used throughout to denote the identity function on $\Gamma, Z(z)=z$ for all $z \in \Gamma$.

LEMMA 1. Suppose $B$ is a uniform algebra on $\Gamma$ such that $\operatorname{Re} A \subset \operatorname{Re} B$. Then there are two points $a, b \in \Gamma \cap\{z: \operatorname{Im} z \geqq 0\}$, 
and a function $\Phi \in B$ such that $\Phi$ maps $\Gamma$ onto itself as in the diagram below. See, also, the notes following diagram.

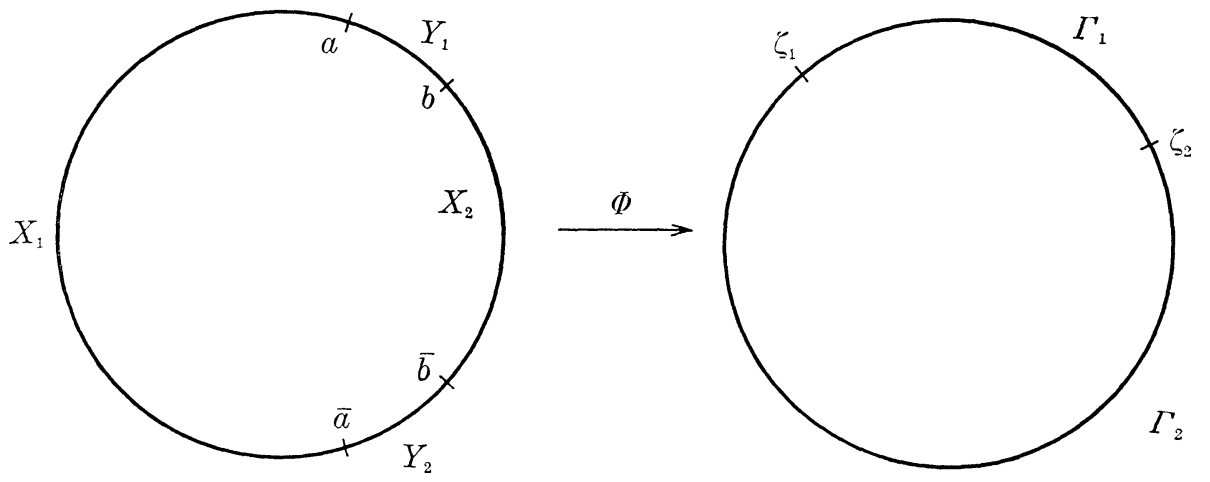

Notes. $X_{1}, X_{2}, Y_{1}$, and $Y_{2}$ are the indicated closed arcs; $\Phi\left(X_{j}\right)$ is a singleton $\left\{\zeta_{j}\right\}$ and $\Phi$ takes $Y_{j}$ homeomorphically onto $\Gamma_{j}$ for $j=1,2$.

Proof. As $Z \in A$, there are functions $\psi$ and $\psi_{1}$ in $B$ such that $\operatorname{Re} Z=\operatorname{Re} \psi$ and $\operatorname{Im} Z=\operatorname{Im} \psi_{1}$. Now if $\psi \operatorname{maps} \Gamma$ onto a Jordan arc, then Mergelyan's theorem implies that the function $w \rightarrow \operatorname{Re} w$ is uniformly approximable on $\psi(\Gamma)$ by polynomials in $\psi$. Since $B$ is a uniformly closed algebra, we have $\operatorname{Re} Z \in B$. Similarly, if also $\psi_{1}$ maps $\Gamma$ onto a Jordan arc, then $\operatorname{Im} Z \in B$. Thus in the event that both $\psi(\Gamma)$ and $\psi_{1}(\Gamma)$ are Jordan arcs, we conclude by Fejér's theorem that $B=C(\Gamma)$. The conclusion of the lemma obviously holds in this case. So let us assume that at least one of $\psi(\Gamma)$ or $\psi_{1}(\Gamma)$, say the former, is not a Jordan arc. Remembering that $\operatorname{Re} \psi=\operatorname{Re} Z$, we see that it is necessarily the case that there exist $a, b \in \Gamma \cap\{z: \operatorname{Im} \mathrm{z} \geqq 0\}$ with $\operatorname{Re} a<\operatorname{Re} b$ such that $\psi(a)=\psi(\bar{a}), \psi(b)=$ $\psi(\bar{b})$, but $\psi(\zeta) \neq \psi(\bar{\zeta})$ for $\operatorname{Re} a<\operatorname{Re} \zeta<\operatorname{Re} b$. Let $X_{1}, X_{2}, Y_{1}, Y_{2}$ be the closed arcs indicated in the diagram. Then $\psi$ maps each of $Y_{1}$ and $Y_{2}$ homeomorphically onto arcs and maps $Y_{1} \cup Y_{2}$ onto a simple closed curve $J$. Let $W$ be the bounded component of $C-J$ and $K$ be the union of $\psi(\Gamma)$ and the bounded components of $C-\psi(\Gamma)$. By a famous theorem of Carathéodory, there is a homeomorphism $\phi$ of $W \cup J$ onto the closed unit disc such that $\phi$ is analytic on $W$; if we extend $\phi$ to $K$ by defining $\phi(w)=\phi(\psi(a))$ for $\operatorname{Re} w<\operatorname{Re} \psi(a)$ and $\dot{\phi}(w)=\phi(\psi(b))$ for $\operatorname{Re} w>\operatorname{Re} \psi(b)$, then $\phi$ is continuous on $K$ and analytic on the interior of $K$. By Mergelyan's theorem, we see that $\dot{\phi}$ is uniformly approximable on $K$ by polynomials and so it follows that $\phi \circ \psi \in B$. The function $\Phi=\phi \circ \psi$ has the required properties. 
For $X \subset \Gamma, B \mid X$ will denote the algebra of restrictions to $X$ of the members of $B$.

LEMMA 2. Let $B$ be a uniform algebra on $\Gamma$ such that $\operatorname{Re} A \subset \operatorname{Re} B$ and let $\Phi \in B$ map $\Gamma$ onto $\Gamma$ as in Lemma 1. Then $B \mid X_{1}=C\left(X_{1}\right)$ and $B \mid X_{2}=C\left(X_{2}\right)$.

Proof. We have $\Phi\left(X_{1}\right)=\left\{\zeta_{1}\right\}$ while $\Phi(\zeta) \in \Gamma-\left\{\zeta_{1}\right\}$ for $\zeta \in \Gamma-X_{1}$. Therefore $X_{1}$ is a peak set for $B$ and consequently $B \mid X_{1}$ is supnorm closed in the space $C\left(X_{1}\right)$. [5, Lemma 12.3.] In fact, an application of the generalized Stone-Weierstrass theorem will show that $B \mid X_{1}=C\left(X_{1}\right)$. For to begin with, it follows from the theory of conformal mapping of Jordan regions (already used once in the proof of Lemma 1) that there is a function $f$ in $A$ which takes $\Gamma$ homeomorphically onto the (boundary of) the closed unit square and is such that $f\left(X_{1}\right)=\{x: 0 \leqq x \leqq 1\}$. We have $\operatorname{Re} A \subset \operatorname{Re} B$, so choose $g \in B$ such that $\operatorname{Re} f=\operatorname{Re} g$. As $\operatorname{Re} f$ is one-to-one on $X_{1}$, so too is Re $g$; thus $g$ maps $X_{1}$ onto a Jordan arc. By Mergelyan's theorem once more, there is a sequence $\left\{P_{n}\right\}$ of polynomials such that $P_{n}(w) \rightarrow$ Re $w$ uniformly on $g\left(X_{1}\right)$; hence $P_{n} \circ g(\zeta) \rightarrow \operatorname{Re} g(\zeta)$ uniformly on $X_{1}$. As $B \mid X_{1}$ is closed we have $\operatorname{Re} g\left|X_{1} \in B\right| X_{1}$. But $\operatorname{Re} g=\operatorname{Re} f$ is one-to-one on $X_{1}$. Thus $B \mid X_{1}$ contains a real-valued function (viz. $\left.\operatorname{Re} g \mid X_{1}\right)$ which is one-to-one. In particular, the real-valued functions in $B \mid X_{1}$ separate the points of $X_{1}$. It follows from the generalized Stone-Weierstrass theorem [5, Theorem 12.1] that $B \mid X_{1}=$ $C\left(X_{1}\right)$ as required. Exactly the same argument applies to show that $B \mid X_{2}=C\left(X_{2}\right)$.

Before turning to the third and final lemma needed for the proof of Theorem 1, let us stop and note how O'Connell's result follows readily from what we have already done. That is, assume we actually have the equality $\operatorname{Re} A=\operatorname{Re} B$. Then $\operatorname{Re} A \mid X_{1}=$ $\operatorname{Re} B \mid X_{1}=C_{R}\left(X_{1}\right)$, the last equation being a consequence of Lemmas 1 and 2. By a result of Sidney and Stout [5, Theorem 20.9] we conclude that $A \mid X_{1}=C\left(X_{1}\right)$. But as is well-known, $A \mid X_{1}=C\left(X_{1}\right)$ only if the "arc" $X_{1}$ is a single point. Of course the same argument yields that $X_{2}$ is a single point. Consequently, (see diagram again) $a=\bar{a}=-1$ and $b=\bar{b}=1$, and hence $\Phi$ is one-to-one. Now $A \circ \Phi \subset$ $B$ implies $A \subset B \circ \Phi^{-1}$, so $B \circ \Phi^{-1}$ is a uniform algebra on $\Gamma$ which lies between $A$ and $C(\Gamma)$. By Wermer's maximality theorem, $B \circ \Phi^{-1}=$ $A$ or $B \circ \Phi^{-1}=C(\Gamma)$. The latter equality is impossible $(\operatorname{Re} A \neq$ $C_{R}(\Gamma)$ ), so $B=A \circ \Phi$ and we have O'Connell's result.

As preparation for Lemma 3, we need to introduce some additional terminology and recall a few facts. Let $m$ denote normalized Lebesgue one-dimensional measure on $\Gamma$ and let $L^{p}(m)$ have its 
usual meaning. $H^{p}(m)$ is the subspace of $L^{p}(m)$ consisting of those $f \in L^{p}(m)$ whose Fourier coefficients with negative indices are zero: thus $f \in H^{p}(m)$ iff $f \in L^{p}(m)$ and $\int_{\Gamma} Z^{n} f d m=0$ for $n=1,2, \cdots$. For $f \in L^{1}(m)$, let $P[f]$ denote the Poisson integral of $f$. It is a basic fact that $P[f]$ is a (complex-valued) harmonic function on $U$ and $P[f]$ is analytic if and only if $f \in H^{1}(m)$. Also, $P[f]$ has radial limits equal to $f$ a.e., and when $f \in H^{1}(m)$, then $f(\zeta)=0$ on a set of positive measure if and only if $P[f]$ is identically zero.

Lemma 3. Suppose $S$ is a subalgebra of $L^{\infty}(m), h \in H^{1}(m)$ with $h \not \equiv 0$, and $h f \in H^{1}(m)$ for each $f \in S$. Then $S \subset H^{\infty}(m)$.

Proof. We can assume that $S$ is closed in $L^{\infty}(m)$ as the closure of $S$ will satisfy the hypothesis if $S$ does. Now for any $f_{1}, f_{2} \in S$, $P[h] \cdot P\left[f_{1} f_{2} h\right]$ and $P\left[f_{1} h\right] \cdot P\left[f_{2} h\right]$ are each of bounded characteristic (belong to the Nevanlinna class $N$, cf. [4], Chapter 17) and, further, have the same radial limits a.e. Thus $\left(P[h] \cdot P\left[f_{1} f_{2} h\right]\right)(z)=\left(P\left[f_{1} h\right]\right.$. $\left.P\left[f_{2} h\right]\right)(z)$ for all $z$ in $U$. This means that for any fixed $z$ where $P[h](z) \neq 0$, the functional

$$
f \longrightarrow \frac{P[f h]}{P[h]}(z) \quad(f \in S)
$$

is a multiplicative linear functional on the Banach algebra $S$; thus it is continuous and has norm $\leqq 1$. Consequently, for each $f \in S$, the meromorphic function $P[f h] / P[h]$ is actually bounded on $U$ and since $P[f h] / P[h]$ has radial limits equal to $f$ a.e., we conclude that $f \in H^{\infty}(m)$. So $S \subset H^{\infty}(m)$ as required.

Proof of Theorem 1. Let $\Phi \in B$ map $\Gamma$ onto $\Gamma$ as in Lemma 1 . We have $B \mid X_{1}=C\left(X_{1}\right)$ and $B \mid X_{2}=C\left(X_{2}\right)$ by Lemma 2. Now let us consider the remaining restriction algebras $B \mid Y_{1}$ and $B \mid Y_{2}$, and the two possibilities: (1) $B \mid Y_{1}=C\left(Y_{1}\right)$ and $B \mid Y_{2}=C\left(Y_{2}\right)$, (2) $B \mid Y_{1} \neq$ $C\left(Y_{1}\right)$ or $B \mid Y_{2} \neq C\left(Y_{2}\right)$. If (1) holds then $B=C(\Gamma)$ because of the following theorem of R. E. Mullins [2, Theorem 3, p. 272]: "Let $A$ be a function algebra on a compact metric space $X$. Let $F_{1}, \cdots, F_{n}$ be $n$ closed sets such that $X=\bigcup_{i=1}^{n} F_{i}$ and $A \mid F_{i}=C\left(F_{i}\right), \quad i=$ $1,2, \cdots, n$. Then $A=C(X)$." (A generalization of Mullins' result can be found in [5], Theorem 13.11.)

Suppose then that (2) holds with say $B \mid Y_{1} \neq C\left(Y_{1}\right)$. By a theorem of Glicksberg [5, Theorem 20.16] there is a complex regular Borel measure $\mu$ on $\Gamma$ such that $\int_{\Gamma} g d \mu=0$ for all $g \in B$, but the restriction $\mu \mid Y_{1}$ is not the zero measure. The same theorem implies, 
moreover, that each of $\mu \mid X_{1}$ and $\mu \mid X_{2}$ is the zero measure. Denote by $\mu^{*}$ the measure on $\Gamma$ induced by $\Phi$; that is, $\mu^{*}(E)=\mu\left(\Phi^{-1}(E)\right)$ for each Borel set $E$. Since $\Phi$ maps $Y_{1}$ homeomorphically onto $\Gamma_{1}$ and $\mu \mid Y_{1}$ is not the zero measure, it follows that $\mu^{*}$ is not the zero measure either. For each $g \in B$, let $g^{*}$ be defined on $\Gamma-\left\{\zeta_{1}, \zeta_{2}\right\}$ by $g^{*}(\zeta)=g\left(\Phi^{-1}(\zeta)\right)$. We claim that $d \mu^{*}=h d m$ where $h \in H^{1}(m)$ and that $g^{*} h \in H^{1}(m)$. To see this let $g \in B$ and $n$ be a positive integer. Then

$$
0=\int_{\Gamma} \Phi^{n} g d \mu=\int_{\Gamma-\left(X_{1} \cup x_{2}\right)} \Phi^{n} g d \mu=\int_{\Gamma-\left\{\zeta_{1}, \zeta_{2}\right\}} Z^{n} g^{*} d \mu^{*}=\int_{\Gamma} Z^{n} g^{*} d \mu^{*} .
$$

If we take $g \equiv 1$, then the preceding calculation, together with the classical F. and M. Riesz theorem, implies that $d \mu^{*}=h d m$ where $h \in H^{1}(m)$. This establishes the claim. Note also that since $\mu^{*}$ is not the zero measure, $h$ cannot vanish on a set of positive $m$-measure. Now let $S$ be the subalgebra of $L^{\infty}(m)$ consisting of $\left\{g^{*}: g \in B\right\}$. Lemma 3 applies and we conclude that $S \subset H^{\infty}(m)$. So $g^{*} \in H^{\infty}(m)$ for each $g \in B$. It is clear that the bounded analytic extension to $U$ of $g^{*}$ is continuous on $U \cup \Gamma-\left\{\zeta_{1}, \zeta_{2}\right\}$ and that, furthermore, $\lim _{\substack{\xi \rightarrow \zeta_{1} \\ \zeta \in \Gamma_{1}-\left\{\zeta_{1}\right\}}} g^{*}(\zeta)=g(a)$ while $\lim _{\zeta \rightarrow \zeta_{1}} g^{*}-\{(\zeta)=g(\bar{a})$. By an old theorem due to E. Lindelöf $[1$, p. 43], we deduce that $g(a)=$ $g(\bar{a})=\lim _{\substack{\zeta \rightarrow \zeta_{1} \\ \zeta \in \bar{U}-\left\{\zeta_{1}\right\}}} g^{*}(\zeta)$. In the same way, $g(b)=g(\bar{b})=\lim _{\substack{\zeta \rightarrow \zeta_{2} \\ \zeta \in \bar{U}-\left\{\zeta_{2}\right\}}} g^{*}(\zeta)$. As $B$ separates points, we conclude that $a=\bar{a}$ and $b=\bar{b}^{\zeta \in \bar{U}-\left\{\zeta_{2}\right\}}$ so that $\Phi$ is one-to-one. The above argument also shows that then $g \circ \Phi^{-1} \in A$ for each $g \in B$. So $B \subset A \circ \Phi$. But we already have $A \circ \Phi \subset B$, hence $A \circ \Phi=B$ and the proof of the theorem is now complete.

REMARK. Although Wermer's maximality theorem was used to give a short proof of O'Connell's result, the above proof of the generalization does not require the maximality theorem. What the proof does use is some of the techniques that appear in Wermer's original argument.

A compact subset $K \subset \Gamma$ is an interpolation set for $A$ if $A \mid K=$ $C(K)$. Say that a continuous map $\Phi: \Gamma \rightarrow \Gamma$ preserves interpolation sets if $A \mid K=C(K)$ implies $A \mid \Phi(K)=C(\Phi(K))$.

Theorem 2. Suppose $\Phi$ is a continuous map of $\Gamma$ into $\Gamma$ such that $\operatorname{Re} A \subset \operatorname{Re} A \circ \Phi$. Then $\Phi$ preserves interpolation sets.

Proof. Let $K$ be an interpolation set for $A$ so that $C(K)=$ $A \mid K$. Then $C_{R}(K)=\operatorname{Re}(A \mid K) \subset \operatorname{Re}(A \circ \Phi \mid K)$. This implies that $C_{R}(\Phi(K)) \subset \operatorname{Re} A \mid \Phi(K)$. Hence by the Sidney-Stout result once more, we conclude that $C(\Phi(K))=A \mid \Phi(K)$ as reguired. 
Note that the preceding simple result is true in the setting of general uniform algebras. It would be interesting to know whether; in general, a map $\Phi$ satisfying the hypothesis of Theorem 2 necessarily preserves peak sets. In certain special cases interpolation sets are automatically peak sets.

Corollary. Let $\Phi$ be a homeomorphism of $\Gamma$ such that $\operatorname{Re} A \subset$ $\operatorname{Re} A \circ \Phi$. Then $\Phi$ is absolutely continuous.

Proof. By Theorem 2, $\Phi$ maps interpolation sets to interpolation sets. But by the Rudin-Carleson theorem these are the closed sets of measure zero. So the homeomorphism $\Phi$ maps closed sets of measure zero to closed sets of measure zero. It follows that $\Phi$ is absolutely continuous.

Although Theorem 1 generalizes in a trivial way to the case where $\Gamma$ is the boundary of an arbitrary Jordan region in the plane, this is the only extension of Theorem 1 that we know of. It would be of interest to determine its validity in some other cases.

Added in proof. Results of E. L. Arenson can be used to show that in the setting of general uniform algebras, a homeomorphism $\Phi$ satisfying the hypothesis of Theorem 2 necessarily preserves peak sets.

\section{REFERENCES}

1. C. Caratheodory, Theory of Functions of a Complex Variable, vol. II, Chelsea Publishing Co., New York, 1960.

2. R. Mullins, The essential set of function algebras, Proc. Amer. Math. Soc., 18 (1967), 271-273.

3. J.M. F. O'Connell, Real parts of uniform algebras, Pacific J. Math., 46 (1973), 235-247.

4. W. Rudin, Real and Complex Analysis, McGraw-Hill, New York, 1966,

5. E. L. Stout, The Theory of Uniform Algebras, Bogden and Quigley, New York, 1971.

Received July 17, 1974 and in revised form August 27, 1974.

Florida State University 


\title{
PACIFIC JOURNAL OF MATHEMATICS
}

\author{
EDITORS
}

RICHARD ARENS (Managing Editor)

University of California

Los Angeles, California 90024

R. A. Beaumont

University of Washington

Seattle, Washington 98105
J. DugundJI

Department of Mathematics University of Southern California Los Angeles, California 90007

D. Gilbarg and J. Milgram

Stanford University

Stanford, California 94305

\section{ASSOCIATE EDITORS}

E. F. BECKENBACH

B. H. NeUManN

F. WOLF

K. YosHIDA

\section{SUPPORTING INSTITUTIONS}

UNIVERSITY OF BRITISH COLUMBIA

GALIFORNIA INSTITUTE OF TECHNOLOGY

UNIVERSITY OF CALIFORNIA

MONTANA STATE UNIVERSITY

UNIVERSITY OF NEVADA

NEW MEXICO STATE UNIVERSITY

OREGON STATE UNIVERSITY

UNIVERSITY OF OREGON

OSAKA UNIVERSITY
UNIVERSITY OF SOUTHERN CALIFORNIA

STANFORD UNIVERSITY

UNIVERSITY OF TOKYO

UNIVERSITY OF UTAH

WASHINGTON STATE UNIVERSITY

UNIVERSITY OF WASHINGTON

AMERICAN MATHEMATICAL SOCIETY NAVAL WEAPONS CENTER 


\section{Pacific Journal of Mathematics}

\section{Vol. 57, No. $1 \quad$ January, 1975}

Keith Roy Allen, Dendritic compactification ......................... 1

Daniel D. Anderson, The Krull intersection theorem ..................... 11

George Phillip Barker and David Hilding Carlson, Cones of diagonally dominant

matrices .............................................

David Wilmot Barnette, Generalized combinatorial cells and facet splitting ........

Stefan Bergman, Bounds for distortion in pseudoconformal mappings ............

Nguyên Phuong Các, On bounded solutions of a strongly nonlinear elliptic

equation ...............................................

Philip Throop Church and James Timourian, Maps with 0-dimensional critical

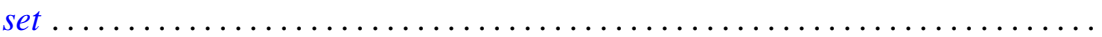

G. Coquet and J. C. Dupin, Sur les convexes ubiquitaires ................. 67

Kandiah Dayanithy, On perturbation of differential operators............... 85

Thomas P. Dence, A Lebesgue decomposition for vector valued additive set

functions .............................................. 91

John Riley Durbin, On locally compact wreath products.................. 99

Allan L. Edelson, The converse to a theorem of Conner and Floyd ............. 109

William Alan Feldman and James Franklin Porter, Compact convergence and the

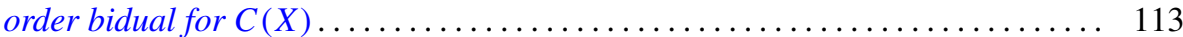

Ralph S. Freese, Ideal lattices of lattices......................... 125

R. Gow, Groups whose irreducible character degrees are ordered by divisibility ... 135

David G. Green, The lattice of congruences on an inverse semigroup ............ 141

John William Green, Completion and semicompletion of Moore spaces .......... 153

David James Hallenbeck, Convex hulls and extreme points of families of starlike and close-to-convex mappings .................................. 167

Israel (Yitzchak) Nathan Herstein, On a theorem of Brauer-Cartan-Hua type ...... 177

Virgil Dwight House, Jr., Countable products of generalized countably compact spaces ............................................ 183

John Sollion Hsia, Spinor norms of local integral rotations. I . . . . . . ......... 199

Hugo Junghenn, Almost periodic compactifications of transformation

semigroups....................................

Shin'ichi Kinoshita, On elementary ideals of projective planes in the 4-sphere and oriented $\Theta$-curves in the 3 -sphere ............................ 217

Ronald Fred Levy, Showering spaces............................ 223

Geoffrey Mason, Two theorems on groups of characteristic 2-type............. 233

Cyril Nasim, An inversion formula for Hankel transform ................... 255

W. P. Novinger, Real parts of uniform algebras on the circle ................ 259

T. Parthasarathy and T. E. S. Raghavan, Equilibria of continuous two-person

games............................................... 265

John Pfaltzgraff and Ted Joe Suffridge, Close-to-starlike holomorphic functions of several variables ....................................... 271

Esther Portnoy, Developable surfaces in hyperbolic space .................. 281

Maxwell Alexander Rosenlicht, Differential extension fields of exponential type . . . 289

Keith William Schrader and James Lewis Thornburg, Sufficient conditions for the

existence of convergent subsequences ........................... 301

Joseph M. Weinstein, Reconstructing colored graphs .................. 307 